\title{
Contribuição para o conhecimento das comunidades anfíbias no sul de Portugal
}

\author{
Marízia Menezes Dias Pereira ${ }^{1}$ e Carlos Silva Neto²
}

Recebido em 7/11/2006. Aceito em 8/10/2007

\begin{abstract}
RESUMO - (Contribuição para o conhecimento das comunidades anfíbias no sul de Portugal). As comunidades anfíbias pioneiras da bacia hidrográfica do Rio Sado (Setor Mariânico-Monchiquense, Subprovíncia Luso-Extremadurense e Setor Ribatagano-Sadense, Subprovíncia Sadense-Divisório Portuguesa) foram objeto do presente estudo. Destacando-se a composição florística de cada fitocenose, bem como os dados referentes à sua ecologia, corologia e sintaxonomia, são apresentadas as principais comunidades vegetais anfíbias identificadas: Junco capitati-Isoetetum hystricis Braun-Blanquet 1936, Junco pygmaei-Isoetetum velati Rivas Goday 1956, Periballio laevis-Illecebretum verticillati Rivas Goday 1954, Loto subbiflori-Chaetopogonetum fasciculati Rivas-Martínez \& Costa in RivasMartínez, Costa, Castroviejo \& E. Valdés 1980, Hyperico elodis-Rhynchosporetum rugosae Neto, Capelo, J.C. Costa \& Lousã in Neto 1997, Anagallido tenellae-Juncetum bulbosi Braun-Blanquet 1967, Utriculario exoletae-Sphagnetum auriculati Neto, Capelo, J.C. Costa \& Lousã 1996 e Cirsio palustris-Juncetum rugosi Neto, Capelo, J.C. Costa \& Lousã 1996). Este estudo mostrou que essas comunidades vegetais são, em sua maioria, relictos, com áreas de distribuição restritas e encontram-se fortemente pressionadas pela crescente ação antrópica e pelas alterações climáticas. Embora com características claramente atlânticas, foram sendo invadidas por espécies mediterrânicas, cujo domínio foi progressivamente aumentando desde o período Atlântico (7800-5700 B.P.), durante o qual se verificou o seu ótimo.
\end{abstract}

Palavras-chave: ecologia de comunidades, plantas anfíbias, sintaxonomia, corologia

\begin{abstract}
Contributions to the knowledge of the amphibian communities in the South of Portugal). The pioneer amphibian communities of the Sado river basin (Marianic-Monchiquensean Sector, Lusitan-Extremadurean and Subprovince Ribatagan-Sadensean Sector, Sadensean-Divinding Portuguese Subprovince) were subject of the present study. The main plant communities were identified (Junco capitati-Isoetetum hystricis Braun-Blanquet 1936, Junco pygmaei-Isoetetum velati Rivas Goday 1956, Periballio laevis-Illecebretum verticillati Rivas Goday 1954, Loto subbiflori-Chaetopogonetum fasciculati Rivas-Martínez \& Costa in Rivas-Martínez, Costa, Castroviejo \& E. Valdés 1980, Hyperico elodis-Rhynchosporetum rugosae Neto, Capelo, J.C. Costa \& Lousã in Neto 1997, Anagallido tenellae-Juncetum bulbosi Braun-Blanquet 1967, Utriculario exoletae-Sphagnetum auriculati Neto, Capelo, J.C. Costa \& Lousã 1996 and Cirsio palustris-Juncetum rugosi Neto, Capelo, J.C. Costa \& Lousã 1996) and its floristic composition presented, as well as other relevant data concerning to ecology, chorology and syntaxonomy of each community. We concluded that most vegetation communities are relict and have restricted distribution areas. The vegetation is under increasing stress due to antropic activities and climate change. This vegetation is dominated by Atlantic communities and species. However, since the Atlantic period (7800-6700 B.P.) the dominance of Mediterranean elements tends to increase.
\end{abstract}

Key words: community ecology, amphibian plants, sintaxonomy, corology

\section{Introdução}

A diversidade das comunidades anfíbias existentes nos charcos temporários tem despertado particular atenção da comunidade científica ibérica para essas áreas. Com o objetivo de proteger e gerir o patrimônio natural, a Comunidade Européia estabeleceu uma política ambiental de preservação dos hábitats da flora e fauna selvagens considerados ameaçados, raros ou vulneráveis. Assim, com a implementação da diretiva comunitária
"Habitats" - Directiva 92/43/CEE do Conselho de 21 de maio de 1992, destacou-se a importância das comunidades anfíbias, concedendo-lhes o status de hábitat prioritário: Charcos temporários mediterrânicos (3170) - Anexo II (relativo à preservação dos hábitats e da fauna e da flora selvagens) do Decreto-Lei n. 140/99, de 24 de abril. Essas comunidades têm em comum a dependência da umidade edáfica - a oscilação da profundidade do lençol freático superficial e o tempo de inundação - e a qualidade da água, que condicionam a

\footnotetext{
1 Universidade de Évora, Colégio Luís António Verney, Departamento de Planeamento Biofísico e Paisagístico, Rua Romão Ramalho 59, 7000 Évora, Portugal

2 Universidade de Lisboa, Departamento de Geografia da Faculdade de Letras, Alameda da Universidade 1600-214 Lisboa, Portugal

3 Autor para correspondência: marizia_cmdp@hotmail.com
} 
distinção dos diferentes biótopos.

No âmbito do projeto "Flora e Vegetação Natural do Alentejo" e com o objetivo de atribuir ou não o status de hábitat prioritário, efetuou-se um estudo nos charcos temporários da bacia hidrográfica do Rio Sado, nomeadamente no Monte das Cabras, em Vila Nova da Baronia ( $38^{\circ} 19^{\prime} \mathrm{N}$ e $\left.8^{\circ} 37^{\prime} \mathrm{W}\right)$, e no Açude da Batalha, Texugueiras, Moinho da Ordem, Açude dos Cachopos e Apostiça em Alcácer do Sal ( $38^{\circ} 21^{\prime} \mathrm{N}$ e $\left.8^{\circ} 39^{\prime} \mathrm{W}\right)$, nos distritos de Évora e Setúbal, da Província do Alto Alentejo.

Litologicamente, os territórios estudados são constituídos por séries cristalofílicas azóicas, areias, arenitos e conglomerados, em um relevo suave ondulado (Barros e Carvalhosa \& Galopim de Carvalho 1969; Zbyszewski et al. 1976). Biogeograficamente, pertencem aos setores Mariânico-Monchiquense e RibataganoSadense (Neto, C.S., dados não publicados; Pereira, M.M.D., dados não publicados) e apresentam um macrobioclima Mediterrânico Pluviestacional oceânico.

Considerados os condicionantes ecológicos mencionados, estudaram-se os seguintes tipos de comunidades higrófilas e herbáceas: 1. Vegetação anfíbia pioneira (Isoeto-Nanojuncetea); 2. Vegetação anfíbia vivaz (Isoeto-Litorelletea); 3. Vegetação de turfeiras planas (Scheuchzerio palustris-Caricetea nigrae); 4. Comunidades higrófilas de Utricularia (Utricularietea intermedio-minoris) e 5. Vegetação vivaz mesofítica (Molinio-Arrhenatheretea).

\section{Material e métodos}

No estudo da vegetação, aplicou-se o método de Braun-Blanquet ou clássico sigmatista (Braun-Blanquet 1979), atualizado por Géhu \& Rivas-Martínez (1981), que se desenvolveu em duas etapas: a analítica e a sintética.

$\mathrm{Na}$ primeira, calcularam-se as áreas mínimas e efetuaram-se os inventários fitossociológicos (ou florísticos) nas estações de Vila Nova da Baronia e Alcácer do Sal. Com base em Braun-Blanquet (1979), as superfícies inventariadas apresentavam uma extensão suficiente para congregar todas as espécies vegetais do hábitat em estudo. Para que a amostragem fosse correta, realizou-se o cálculo da "área mínima" para os diferentes tipos de vegetação, em cada uma das estações. Em primeiro lugar, definiu-se uma área-base (p.ex. um quadrado com $40 \mathrm{~cm}$ de lado), anotando-se todos os taxa vegetais presentes. Em seguida, duplicou-se contiguamente a área anterior e acrescentaram-se ao elenco florístico inicial os novos taxa. Procedeu-se com igual critério às duplicações sucessivas e às anotações de novas presenças, até o ponto em que, duplicando a última área, novas espécies deixaram de aparecer. A área correspondente a tal ponto (área mínima) foi adotada para os inventários florísticos com o mesmo tipo de vegetação, salvo algumas exceções, tais como as dimensões da área encharcada (Pereira 2003).

O inventário florístico consistiu numa ficha na qual se apontaram as informações acerca do habitat: altitude média do local, área mínima adotada e grau de cobertura do solo, que, segundo Guinochet (1973), deve corresponder à superfície coberta pela vegetação, projetado, sobre o solo, o conjunto de todos os indivíduos da área estudada. Fez-se também a inscrição de todos os taxa presentes na área mínima e, para cada um, foram anotados os índices de quantidade e sociabilidade.

Os valores do primeiro resultaram da combinação da abundância e dominância, traduzidos, num só índice, o número de taxa por unidade de superfície e a respectiva dominância. Para facilitar a sua atribuição no inventário florístico, aplicou-se a escala de cobertura para cada taxa: 5 (mais de $75 \%$ da área), 4 (de 50 a $75 \%$ da área), 3 (de 25 a $50 \%$ da área), 2 (de 5 a $25 \%$ da área), 1 (menos de $5 \%$ da área) e + (indivíduos raros ou muito raros na área) (Pereira 2003). Na sociabilidade, avaliouse a forma como as plantas da mesma espécie estão associadas entre si, expressando-se pelos índices: 5 (povoamentos densos), 4 (pequenas colônias ou tapetes), 3 (pequenas manchas), 2 (grupo ou grupos) e 1 (indivíduos isolados) (Pereira 2003).

Na etapa sintética, os inventários selecionados foram reunidos em quadros, de acordo com as afinidades florísticas. Nos cabeçalhos, foram inscritas as identificações de cada inventário florístico: número de ordem, altitude média (m.s.m), área mínima $\left(\mathrm{m}^{2}\right)$ e grau de cobertura (\%). Nas linhas horizontais, foram apontados todos os taxa e, nas colunas verticais, os respectivos índices de quantidade e sociabilidade, por ordem decrescente de presença. Em primeiro lugar, aparece o grupo das espécies características da associação e das unidades superiores (aliança, ordem e classe) e, em segundo, no das "outras espécies", os taxa pertencentes a outras unidades taxonômicas (Pereira 2003). A fim de evitar o uso de trinômios nos quadros fitossociológicos, abreviaram-se os taxa, ficando, por exemplo: Ranunculus aleae (Ranunculus bulbosus L. subsp. aleae (Willk.) Rouy \& Fouc.), Lotus subbiflorus (Lotus subbiflorus Lag. subsp. subbiflorus) e Myosotis caespitosa (Myosotis laxa Lehm. subsp. caespitosa (C.F. Schultz) Hyl), entre outros.

A metodologia clássica sigmatista também permitiu definir, limitar e hierarquizar as unidades fitossociológicas. A associação, que é a unidade básica da Fitossociologia, está definida por um conjunto de indivíduos que têm em comum as características florísticas, ecológicas, 
dinâmicas e corológicas (Braun-Blanquet 1979). Estes apresentam afinidades entre si, o que permite agrupá-los em unidades taxonômicas inferiores (associações) e superiores (alianças, ordens e classes). A unidade imediatamente superior é a aliança, que reúne as associações semelhantes do ponto de vista ecológico, assinaladas por espécies características dessa unidade taxonômica. As alianças florísticas e ecologicamente próximas são reunidas em ordens, que têm amplitudes ecológicas grandes e só se modificam quando há alterações profundas no hábitat. Finalmente, a classe reúne as ordens floristicamente semelhantes. As espécies dessa unidade taxonômica têm grandes amplitudes ecológicas e nela estão incluídas as espécies características das ordens, alianças e associações (Pereira 2003).

$\mathrm{Na}$ nomenclatura das unidades hierárquicas, seguiu-se o Código de Nomenclatura Fitossociológica que está compilado na obra de Barkman et al. (1988) e em Izco \& Del Arco (2003). Por princípio, aceitou-se a escala de unidades de base florística com os respectivos sufixos latinos, que se associaram às iniciais de espécie dominante ou sociologicamente importante: classe (etea), ordem (etalia), aliança (ion) e associação (etum).

\section{Resultados e discussão}

Com base em Rivas-Martínez et al. (2001; 2002), foram analisadas cinco classes fitossociológicas: IsoetoNanojuncetea Braun-Blanquet \& Tüxen ex Westhoff, Dijk \& Passchier 1946; Isoeto-Littorelletea Braun-Blanquet \& Vlieger in Vlieger 1937; Scheuchzerio palustrisCaricetea nigrae Tüxen 1937 nom. mut. propos.; Utricularietea intermedio-minoris Pietsch 1965 e Molinio-Arrhenatheretea Tüxen 1937, nas quais foi possível identificar oito associações, ocorrendo nas estações de Vila Nova da Baronia (Monte das Cabras) e Alcácer do Sal (Açude da Batalha, Texugueiras, Moinho da Ordem, Açude dos Cachopos e Apostiça).

Foram amostradas, nas oito associações, 81 espécies, pertencentes a 28 famílias (Tab.1).

Do elenco florístico, destacaram-se as espécies endêmicas e as insetívoras que apresentam adaptações especiais pouco comuns.

O helófito Hypericum elodes (Guttiferae), é considerado como endêmico da Europa (Franco 1971; Tutin et al. 1968), embora Valdés et al. (1987) lhe atribua a distribuição macaronésica. É uma espécie frequiente em terrenos úmidos ou águas superficiais, em Portugal continental e no Arquipélago dos Açores (Franco 1971). A composta Pulicaria paludosa aparece com freqüência nos sítios úmidos ou temporariamente inundados. Apesar de Franco (1984) considerá-la como endemismo europeu e Tutin et al. (1976) como ibérica, Valdés et al. (1987) registrou a ocorrência desta espécie para a Península Ibérica e Noroeste de África. Juncus acutiflorus subsp. rugosus é uma juncácea rizomatosa endêmica ibérica (Valdés et al. 1987; Franco \& Afonso 2003) e encontrase no Centro e Sul de Portugal continental (Franco \& Afonso 2003), em locais úmidos, prados e margens dos cursos de água. A espécie endêmica ibérica Serapias perez-chiscanoi C. Acedo (Aedo \& Herrero 2005) é uma orquídea rara e encontra-se muito localizada no sul do território português, em prados úmidos, sobre solos silicosos e arenosos.

As insetívoras pertencem às famílias Droseraceae (Drosera intermedia) e Lentibulariaceae (Pinguicula lusitanica, Utricularia gibba. subsp. exoleta e Utricularia australis). Drosera intermedia, conhecida vulgarmente por orvalhinha, rorela e rorela-de-folhascompridas, é um hemicriptófito helófito e gregário. Apresenta folhas basilares cujas páginas superiores estão cobertas por pêlos compridos, vermelhos, glandulares, pegajosos e irritáveis (Franco 1971), que se dobram rapidamente para o centro, afogando qualquer inseto aprisionado nos pêlos. Esta insetívora cresce em prados inundados e turfeiras de esfagno, em solos ácidos (Castroviejo et al. 1993) e arenosos. Encontra-se distribuída pelo Norte da América, Centro e Oeste da Europa e Ásia Menor. Na Península Ibérica aparece na fachada atlântica entre a Galicia espanhola e a Estremadura portuguesa (Castroviejo et al. 1993). Pinguicula lusitanica prefere turfeiras ácidas e charnecas úmidas (Franco 1984). É um hemicriptófito com as folhas alternas ou em roseta basilar e que desenvolveu um método de captura semelhante ao de Drosera intermedia. Nas folhas carnudas, freqüentemente dimórficas, distinguem-se uns pêlos compridos destinados à captura das presas e outros mais curtos, portadores de glândulas digestivas. De acordo com Valdés et al. (1987), esta insetívora encontra-se distribuída pelo Oeste da Europa e Noroeste de África e em Portugal aparece do Minho ao Alentejo litoral (Franco 1971). O gênero Utricularia L. é predominantemente tropical, mas os taxa Utricularia gibba subsp. exoleta e Utricularia australis são espontâneos na Europa e atípicos na formação de gemas no inverno (Franco 1984). Trata-se de plantas aquáticas submersas, em que as folhas filiformes estão providas de pequenas ascídias (urnas) operculadas, cujas aberturas estão rodeadas de pêlos que segregam uma mucosidade que atrai os diminutos animais aquáticos. Utricularia gibba subsp. exoleta coloniza águas superficiais ou vasa líquida (Franco 1984), em solos arenosos na Península Ibérica, Noroeste da África, Sul da Ásia e Austrália (Valdés et al. 1987). Utricularia australis prefere águas estagnadas (Franco 1984), em solos arenosos no continente europeu (Valdés et al. 1987) 
Tabela 1. Composição florística de comunidades vegetais anfíbias da bacia do rio Sado, Portugal. A:_Associação Junco capitati-Isoetetum hystrici; B: Associação Junco pygmaei-Isoetetum velati; C: Associação Periballio laevis-Illecebretum verticillati; D: Associação Loto subbifloriChaetopogonetum fasciculati; E: Associação Hyperico elodis-Rhynchosporetum rugosae; F: Associação Anagallido tenellae-Juncetum bulbosi; G: Associação Utriculario exoletae-Sphagnetum auriculati; H: Associação Cirsio palustris-Juncetum rugosi; N: número de associações em que a espécie foi registrada.

\begin{tabular}{|c|c|c|c|c|c|c|c|c|c|}
\hline Família/Espécie & A & $\mathrm{B}$ & $\mathrm{C}$ & $\mathrm{D}$ & $\mathrm{E}$ & $\mathrm{F}$ & $\mathrm{G}$ & $\mathrm{H}$ & $\mathrm{N}$ \\
\hline \multicolumn{10}{|l|}{ ALISMATACEAE } \\
\hline Alisma plantago-aquatica $\mathrm{L}$. & & & & & $\mathrm{x}$ & & & & 1 \\
\hline Baldellia ranunculoides (L.) Parl. & $\mathrm{x}$ & & & & $\mathrm{x}$ & & & & 2 \\
\hline \multicolumn{10}{|l|}{ APIACEAE } \\
\hline Hydrocotyle vulgaris $\mathrm{L}$. & & & & & $\mathrm{x}$ & & & $\mathrm{x}$ & 1 \\
\hline Oenanthe lachenalii C.C. Gmelin & & & & & & & & $\mathrm{x}$ & 1 \\
\hline \multicolumn{10}{|l|}{ ASTERACEAE } \\
\hline Cirsium palustre (L.) Scop. & & & & & & & & $\mathrm{x}$ & 1 \\
\hline Pulicaria paludosa Link & & & & $\mathrm{x}$ & & & & & 1 \\
\hline \multicolumn{10}{|l|}{ BORAGINACEAE } \\
\hline Myosotis laxa Lehm. subsp. caespitosa (C. F. Schultz) Hyl & & & & $\mathrm{x}$ & & & & & 1 \\
\hline M. welwitschii Boiss. \& Reuter & & $\mathrm{x}$ & & & $\mathrm{x}$ & & & & 2 \\
\hline \multicolumn{10}{|l|}{ CAMPANULACEAE } \\
\hline Lobelia urens L. & & & & & $\mathrm{x}$ & $\mathrm{x}$ & & & 2 \\
\hline Solenopsis laurentia (L.) C. Presl & $\mathrm{x}$ & $\mathrm{x}$ & & $\mathrm{x}$ & $\mathrm{x}$ & & & & 4 \\
\hline \multicolumn{10}{|l|}{ CARYOPHYLLACEAE } \\
\hline Illecebrum verticillatum $\mathrm{L}$. & & $\mathrm{x}$ & $\mathrm{x}$ & $\mathrm{x}$ & & & & & 3 \\
\hline Silene laeta (Aiton) Godron & & & & $\mathrm{x}$ & & & & & 1 \\
\hline \multicolumn{10}{|l|}{ CLUSIACEAE } \\
\hline Hypericum elodes L. & $\mathrm{x}$ & & & & $\mathrm{x}$ & $\mathrm{x}$ & $\mathrm{x}$ & $\mathrm{x}$ & 5 \\
\hline H. humifusum L. & $\mathrm{x}$ & $\mathrm{x}$ & & $\mathrm{x}$ & & & $\mathrm{x}$ & & 4 \\
\hline H. undulatum Schousboe & & & & & $\mathrm{x}$ & $\mathrm{x}$ & & & 2 \\
\hline \multicolumn{10}{|l|}{ CYPERACEAE } \\
\hline Carex demissa Hornem. & $\mathrm{x}$ & & & & $\mathrm{x}$ & $\mathrm{x}$ & $\mathrm{x}$ & & 4 \\
\hline C. lamprocarpa Celak. & & & & & & & & $\mathrm{x}$ & 1 \\
\hline C. lusitanica (Willd.) Maire & & & & & & & & $\mathrm{x}$ & 1 \\
\hline Eleocharis multicaulis (Sm.) Desv. & $\mathrm{x}$ & & & & $\mathrm{x}$ & $\mathrm{x}$ & $\mathrm{x}$ & $\mathrm{x}$ & 5 \\
\hline Fuirena pubescens (Poir.) Kunth & $\mathrm{x}$ & & & & $\mathrm{x}$ & $\mathrm{x}$ & $\mathrm{x}$ & $\mathrm{x}$ & 5 \\
\hline Isolepis cernиa (Vahl) Roemer \& Schultes & & $\mathrm{x}$ & & $\mathrm{x}$ & & & & & 2 \\
\hline I. pseudosetaceus (Dav.) Vasc. & & $\mathrm{x}$ & & $\mathrm{x}$ & & & & & 2 \\
\hline Rhynchospora rugosa (Vahl) S. Gale & & & & & $\mathrm{x}$ & $\mathrm{x}$ & & $\mathrm{x}$ & 3 \\
\hline Schoenoplectus mucronatus (L.) Palla & & & & & & & & $\mathrm{x}$ & 1 \\
\hline Schoenus nigricans L. & & & & & $\mathrm{x}$ & $\mathrm{x}$ & $\mathrm{x}$ & $\mathrm{x}$ & 4 \\
\hline \multicolumn{10}{|l|}{ DROSERACEAE } \\
\hline Drosera intermedia Hayne & $\mathrm{x}$ & & & & $\mathrm{x}$ & $\mathrm{x}$ & $\mathrm{x}$ & & 4 \\
\hline \multicolumn{10}{|l|}{ FABACEAE } \\
\hline Genista anglica $\mathrm{L}$. & & & & & & & & $\mathrm{x}$ & 1 \\
\hline Lotus pedunculatus Cav. & & & & & & & & $\mathrm{x}$ & 1 \\
\hline L. subbiflorus Lag. subsp. subbiflorus & & $\mathrm{x}$ & $\mathrm{x}$ & $\mathrm{x}$ & & & & & 3 \\
\hline L. uliginosus Schkuhr & & & & & & & & $\mathrm{x}$ & 1 \\
\hline Ulex minor Roth & & & & & & & & $\mathrm{x}$ & 1 \\
\hline \multicolumn{10}{|l|}{ GENTIANACEAE } \\
\hline Centaurium maritimum (L.) Fritsch & & $\mathrm{x}$ & $\mathrm{x}$ & & & & & & 2 \\
\hline Cicendia filiformis (L.) Delarbre & $\mathrm{x}$ & $\mathrm{x}$ & & $\mathrm{x}$ & $\mathrm{x}$ & $\mathrm{x}$ & & & 5 \\
\hline Exaculum pusillum (Lam.) Caruel & & $\mathrm{x}$ & & $\mathrm{x}$ & & & & & 2 \\
\hline \multicolumn{10}{|l|}{ ISOETACEAE } \\
\hline Isoetes hystrix Bory & $\mathrm{x}$ & & & & & $\mathrm{x}$ & $\mathrm{x}$ & & 3 \\
\hline I. setaceum Lam. & & & & & & & $\mathrm{x}$ & $\mathrm{x}$ & 2 \\
\hline I. vellata A. Braun & & $\mathrm{x}$ & & & & & & & 1 \\
\hline \multicolumn{10}{|l|}{ JUNCACEAE } \\
\hline Juncus acutiflorus Ehrh. subsp. rugosus (Steudel) Coutinho & & & & & $\mathrm{x}$ & & & & 1 \\
\hline J. bufonius L. & $\mathrm{x}$ & $\mathrm{x}$ & & $\mathrm{x}$ & $\mathrm{x}$ & $\mathrm{x}$ & & & 5 \\
\hline J. capitatus Weigel. & & $\mathrm{x}$ & $\mathrm{x}$ & $\mathrm{x}$ & & & & & 3 \\
\hline J. effusus L. & & & & & & & & $\mathrm{x}$ & 1 \\
\hline J. bulbosus L. var. foliosus Desf & $\mathrm{x}$ & & & & $\mathrm{x}$ & $\mathrm{x}$ & $\mathrm{x}$ & & 4 \\
\hline J.s hybridus Brot. & & $\mathrm{x}$ & & $\mathrm{x}$ & & & & & 2 \\
\hline J. pygmaeus L.C.M. Richard & & $\mathrm{x}$ & & $\mathrm{x}$ & & & & & 2 \\
\hline
\end{tabular}


Tabela 1 (continuação)

\begin{tabular}{|c|c|c|c|c|c|c|c|c|c|}
\hline Família/Espécie & A & $\mathrm{B}$ & $\mathrm{C}$ & $\mathrm{D}$ & $\mathrm{E}$ & $\mathrm{F}$ & G & $\mathrm{H}$ & $\mathrm{N}$ \\
\hline Juncus rugosus (Steudel) Coutinho & $\mathrm{x}$ & & & & $\mathrm{x}$ & $\mathrm{x}$ & $\mathrm{x}$ & $\mathrm{x}$ & 5 \\
\hline J. sorrentinii Parl. & & & & & & & $\mathrm{x}$ & & 1 \\
\hline J. tenageia Ehrh. & $\mathrm{x}$ & $\mathrm{x}$ & & $\mathrm{x}$ & $\mathrm{x}$ & & & & 4 \\
\hline \multicolumn{10}{|l|}{ LAMIACEAE } \\
\hline Lycopus europaeus L. & & & & & $\mathrm{x}$ & & & & 1 \\
\hline \multicolumn{10}{|l|}{ LENTIBULARIACEAE } \\
\hline Pinguicula lusitanica $\mathrm{L}$. & & $\mathrm{x}$ & $\mathrm{x}$ & $\mathrm{x}$ & & $\mathrm{x}$ & $\mathrm{x}$ & & 5 \\
\hline Utricularia australis $\mathrm{R} . \mathrm{Br}$. & & & & & & & $\mathrm{x}$ & & 1 \\
\hline Utricularia gibba L. subsp. exoleta (R.Br. ) P. Taylor & $\mathrm{x}$ & & & & $\mathrm{x}$ & & $\mathrm{x}$ & & 3 \\
\hline \multicolumn{10}{|l|}{ LINACEAE } \\
\hline Radiola linoides Roth & & $\mathrm{x}$ & & $\mathrm{x}$ & & & & & 2 \\
\hline \multicolumn{10}{|l|}{ LYTHRACEAE } \\
\hline Lythrum hyssopifolia $\mathrm{L}$. & & $\mathrm{x}$ & & & & & & & 1 \\
\hline L. junceum Banks \& Solander & & & & & & & & $\mathrm{x}$ & 1 \\
\hline L. salicaria $\mathrm{L}$. & & & & & & & & $\mathrm{x}$ & 1 \\
\hline \multicolumn{10}{|l|}{ MYRICACEAE } \\
\hline Myrica galle $\mathrm{L}$. & & & & & & $\mathrm{x}$ & & & 1 \\
\hline \multicolumn{10}{|l|}{ ONAGRACEAE } \\
\hline Ludwigia palustris (L.) Elliot & & & & & $\mathrm{x}$ & & & & 1 \\
\hline \multicolumn{10}{|l|}{ ORCHIDACEAE } \\
\hline Serapias strictiflora $\mathrm{L}$. & & $\mathrm{x}$ & & & & & & & 1 \\
\hline S. cordigera $\mathrm{L}$. & & & & $\mathrm{x}$ & & & & & 1 \\
\hline S. lingua $\mathrm{L}$. & & & & $\mathrm{x}$ & & & & & 1 \\
\hline S. perez-chiscanoi C. Acedo & & & & $\mathrm{x}$ & & & & & 1 \\
\hline S. strictiflora $\mathrm{L}$. & & & $\mathrm{x}$ & $\mathrm{x}$ & & & & & 2 \\
\hline \multicolumn{10}{|l|}{ POACEAE } \\
\hline Agrostis stolonifera L. & $\mathrm{x}$ & & & & $\mathrm{x}$ & $\mathrm{x}$ & $\mathrm{x}$ & & 4 \\
\hline Chaetopogon fasciculatus (Link) Hayek & & $\mathrm{x}$ & $\mathrm{x}$ & $\mathrm{x}$ & & & & & 3 \\
\hline Festuca arundinacea Schreber & & & & & & & & $\mathrm{x}$ & 1 \\
\hline Holcus lanatus L. & & & & & & & & $\mathrm{x}$ & 1 \\
\hline Molineriella laevis (Brot.) Rouy & & & $\mathrm{x}$ & & & & & & 1 \\
\hline $\begin{array}{l}\text { Molinia caerulea (L.) Moench subsp. arundinacea } \\
\quad \text { (Schrank) K. Richter }\end{array}$ & & & & $\mathrm{x}$ & $\mathrm{x}$ & $\mathrm{x}$ & $\mathrm{x}$ & 4 & \\
\hline Panicum repens $\mathrm{L}$. & & & & & $\mathrm{x}$ & & & & 1 \\
\hline Phragmites australis (Cav.) Steudel & & & & & & & & $\mathrm{x}$ & 1 \\
\hline \multicolumn{10}{|l|}{ POTAMOGETONACEAE } \\
\hline Potamogeton natans L. & & & & & & & $\mathrm{x}$ & & 1 \\
\hline \multicolumn{10}{|l|}{ PRIMULACEAE } \\
\hline Anagallis tenella (L.) L. & & & & & $\mathrm{x}$ & $\mathrm{x}$ & $\mathrm{x}$ & $\mathrm{x}$ & 4 \\
\hline \multicolumn{10}{|l|}{ RANUNCULACEAE } \\
\hline Ranunculus bulbosus L. subsp. aleae (Willk.) Rouy \& Fouc & & $\mathrm{x}$ & & & & & & & 1 \\
\hline R. trilobus Desf. & & & & $\mathrm{x}$ & & & & & 1 \\
\hline \multicolumn{10}{|l|}{ ROSACEAE } \\
\hline Potentilla erecta (L.) Räuschel & & & & & & & $\mathrm{x}$ & $\mathrm{x}$ & 2 \\
\hline Rubus ulmifolius Schott & & & & & & & & $\mathrm{x}$ & 1 \\
\hline \multicolumn{10}{|l|}{ RUBIACEAE } \\
\hline Galium palustre L. & & & & & & & & $\mathrm{x}$ & 1 \\
\hline \multicolumn{10}{|l|}{ SCROPHULARIACEAE } \\
\hline Kickxia cirrhosa (L.) Fritsch & & & & $\mathrm{x}$ & & & & & 1 \\
\hline Parentucellia latifolia (L.) Caruel & $\mathrm{x}$ & & & & & & & & 1 \\
\hline Pedicularis lusitanica Hoffmanns. \& Link & & & & & & $\mathrm{x}$ & & & 1 \\
\hline \multicolumn{10}{|l|}{ SPHAGNACEAE } \\
\hline Sphagnum auriculatum Schimp. & & & & & $\mathrm{x}$ & $\mathrm{x}$ & $\mathrm{x}$ & $\mathrm{x}$ & 4 \\
\hline
\end{tabular}

e de norte a sul do território português (Franco 1971). As associações identificadas são descritas a seguir:

1. Vegetação anfíbia pioneira: classe IsoetoNanojuncetea Braun-Blanquet \& Tüxen ex Westhoff, Dijk \& Passchier 1946
Diz respeito à vegetação de charcos temporários, de terófitos e criptófitos efêmeros de pequeno porte, pioneiros em geral, que se desenvolvem em solos periodicamente encharcados ou alagados com água doce, oligotrófica e eutrófica (Pereira, M.M.D., dados não 
publicados). Alcança o ótimo na Região Mediterrânica, estendendo-se também para a Sub-Região Atlântica Centroeuropéia (Loidi et al. 1997) e, segundo Brullo \& Minissale (1998), a distribuição é euro-mediterrânica e macaronésica. Ainda com base em Rivas-Martínez et al. (2002), identificou-se a ordem Isoetetalia BraunBlanquet 1936, que agrupa comunidades termófilas ou sub-termófilas, cuja biogeografia é, de acordo com Loidi et al. (1997), mediterrânica e ocidental européia e, segundo Brullo \& Minissale (1998), mediterrânica e mediterrânica-subatlântica. Esta unidade apresentou, na área estudada, três alianças: Menthion cervinae BraunBlanquet ex Moor 1937 nom. mut. propos., que reúne as associações tipicamente mediterrânicas (Brullo \& Minissale 1998), Agrostion salmanticae Rivas Goday 1958, de caráter mediterrânico iberoatlântico (Brullo \& Minissale 1998), e Cicendion (Rivas Goday in Rivas Goday \& Borja 1961) Braun-Blanquet 1967, de ótimo iberoatlântico (Loidi et al. 1997).

\subsection{Associação Junco capitati-Isoetetum hystricis Braun-Blanquet 1936}

Esta unidade mostrou-se típica de solos pouco profundos, de hidromorfia variável e que secavam durante o período estival. No Moinho da Ordem, em Alcácer do Sal, predominavam nesta associação as espécies pioneiras de floração primaveril, tais como Isoetes hystrix, Cicendia filiformis e Juncus tenageia (Tab. 2). Insere-se na aliança Menthion cervinae Braun-Blanquet ex Moor 1937 nom. mut. propos.

\subsection{Associação Junco pygmaei-Isoetetum velati Rivas Goday 1957}

Apresentou-se dominada por espécies pioneiras características de depressões mais ou menos profundas, em solos areno-limosos de charcos ou ribeiras temporariamente inundadas, pertencentes à aliança Menthion cervinae Braun-Blanquet ex Moor 1937 nom. mut. propos.. Esta associação foi identificada no Monte das Cabras, em Vila Nova da Baronia (Tab. 3), sendo constituída por Isoetes vellata, Isolepis cernua, Juncus bufonius, Illecebrum verticillatum e Juncus pygmaeus, que florescem no período primaveril-estival.

\subsection{Associação Periballio laevis-Illecebretum verticillati Rivas Goday 1954}

Esta associação caracterizou-se pela presença de comunidades de terófitos de pequeno porte, mesomediterrânicos, com preferência por solos oligotróficos, siliciosos, de origem granítica e quartzítica, pobres em nutrientes (Pereira 2002), revestindo pequenos charcos de pouca profundidade no Inverno e início da Primavera, secando totalmente no Verão. No Monte das Cabras, em
Tabela 2. Junco capitati-Isoetetum hystricis.

\begin{tabular}{|c|c|c|c|c|}
\hline Número de ordem & 1 & 2 & 3 & 4 \\
\hline Altitude média (m) & 22 & 22 & 22 & 22 \\
\hline Área mínima $\left(\mathrm{m}^{2}\right)$ & 0,5 & 0,5 & 1 & 1 \\
\hline Grau de cobertura $(\%)$ & 70 & 90 & 90 & 90 \\
\hline \multicolumn{5}{|c|}{ Características da associação e das unidades superiores } \\
\hline Isoetes hystrix & 2.2 & 2.2 & 2.2 & 1.2 \\
\hline Cicendia filiformis & 1.1 & 1.1 & 1.2 & 1.2 \\
\hline Juncus tenageia & - & - & 2.2 & 2.2 \\
\hline Juncus bufonius & 1.2 & - & - & - \\
\hline Hypericum humifusum & - & 1.1 & - & - \\
\hline Solenopsis laurentia & - & - & - & 1.1 \\
\hline \multicolumn{5}{|c|}{ Outras espécies } \\
\hline Eleocharis multicaulis & 1.2 & 1.2 & 1.2 & 1.2 \\
\hline Hypericum elodes & 2.1 & - & 1.2 & 2.2 \\
\hline Fuirena pubescens & +.2 & - & 1.2 & 1.2 \\
\hline Utricularia exoleta & 2.2 & 2.3 & - & - \\
\hline Juncus foliosus & 2.2 & - & - & 2.2 \\
\hline Drosera intermedia & 1.1 & - & - & - \\
\hline Agrostis stolonifera & +.1 & - & - & - \\
\hline Carex demissa & - & 1.2 & - & - \\
\hline Juncus rugosus & - & - & 2.2 & - \\
\hline Baldellia ranunculoides & - & - & 1.2 & - \\
\hline Parentucellia latifolia & - & - & +.2 & - \\
\hline
\end{tabular}

Locais: 1 a 4 Moinho da Ordem (Alcácer do Sal).

Tabela 3. Junco pygmaei-Isoetetum velati.

\begin{tabular}{|c|c|c|c|c|}
\hline Número de inventário & 1 & 2 & 3 & 4 \\
\hline Altitude média (m) & 270 & 270 & 270 & 270 \\
\hline Área mínima $\left(\mathrm{m}^{2}\right)$ & 0,5 & 1 & 0,5 & 1 \\
\hline Grau de cobertura $(\%)$ & 100 & 100 & 70 & 100 \\
\hline \multicolumn{5}{|c|}{ Características da associação e das unidades superiores } \\
\hline Isoetes velata & 3.3 & 3.2 & 3.3 & 2.2 \\
\hline Isolepis cernua & 3.3 & 1.2 & 1.2 & 1.2 \\
\hline Juncus bufonius & 2.2 & 2.2 & 1.2 & 2.2 \\
\hline Illecebrum verticillatum & 1.2 & 1.2 & 1.2 & 2.3 \\
\hline Juncus pygmaeus & 2.2 & 3.3 & 2.2 & 1.2 \\
\hline Solenopsis laurentia & 2.2 & 1.2 & - & 1.1 \\
\hline Juncus capitatus & 1.2 & 2.2 & - & 2.2 \\
\hline Chaetopogon fasciculatus & 1.2 & 1.2 & 2.2 & - \\
\hline Cicendia filiformis & 1.2 & 1.2 & 1.2 & - \\
\hline Radiola linoides & 1.2 & 1.1 & +.1 & - \\
\hline Juncus tenageia & 1.2 & 1.2 & - & 1.2 \\
\hline Isolepsis pseudosetacea & - & 1.2 & - & 2.2 \\
\hline Hypericum humifusum & - & 1.2 & - & 1.2 \\
\hline Exaculum pusillum & - & 1.1 & - & 1.1 \\
\hline Lotus subbiflorus & - & 1.2 & - & - \\
\hline Juncus hybridus & - & 1.2 & - & - \\
\hline Centaurium maritimum & - & - & - & 1.1 \\
\hline Lythrum hyssopifolia & - & - & - & 1.1 \\
\hline \multicolumn{5}{|c|}{ Outras espécies } \\
\hline Pinguicula lusitanica & 2.2 & 2.3 & 2.2 & 1.1 \\
\hline Serapias strictiflora & 1.2 & 1.2 & 1.1 & - \\
\hline Myosotis welwitschii & +.1 & 1.1 & +.1 & - \\
\hline Ranunculus aleae & - & +.1 & - & +.1 \\
\hline
\end{tabular}

Locais: 1 a 5 Monte das Cabras (Vila Nova da Baronia). 
Vila Nova da Baronia (Tab. 4), esta associação apresentou-se dominada por Illecebrum verticillatum, Lotus subbiflorus subsp. subbiflorus e Chaetopogon fasciculatus. Pertence à aliança Agrostion salmanticae Rivas Goday 1958.

Tabela 4. Periballio laevis-Illecebretum verticillati.

\begin{tabular}{lccc}
\hline Número de ordem & 1 & 2 & 3 \\
\hline Altitude média (m) & 270 & 270 & 270 \\
Área mínima (m²) & 4 & 4 & 4 \\
Grau de cobertura (\%) & 100 & 90 & 80 \\
\multicolumn{4}{c}{ Características da associação e das unidades superiores } \\
Illecebrum verticillatum & 2.3 & 1.2 & 2.2 \\
Lotus subbiflorus & 1.1 & 1.2 & 1.1 \\
Chaetopogon fasciculatus & 1.2 & 1.2 & - \\
Centaurium maritimum & - & 2.1 & - \\
Juncus capitatus & - & - & 1.2 \\
\multicolumn{1}{c}{ Molineriella laevis } & Outras espécies & & \\
Serapias strictiflora & 2.2 & 2.2 & 1.2 \\
Pinguicula lusitanica & 2.2 & 1.2 & 2.2 \\
Silene laeta & +.1 & +.2 & +.1 \\
& 1.2 & - & 1.1 \\
\hline
\end{tabular}

Locais: 1 a 3 Monte das Cabras (Vila Nova da Baronia).

\subsection{Associação Loto subbiflori-Chaetopogonetum}

fasciculati Rivas-Martínez \& Costa in RivasMartínez, Costa, Castroviejo \& E. Valdés 1980

Foi identificada nos prados de solos encharcados termo-mesomediterrânicos, de aspecto graminóide, constituídos por terófitos efêmeros de pouca biomassa e que revestiam áreas depressionárias durante o Inverno e início da Primavera, em solos areno-limosos. No Monte das Cabras, em Vila Nova da Baronia (Tab. 5), predominavam Juncus bufonius, Chaetopogon fasciculatus, Solenopsis laurentia, Kickxia cirrhosa e Illecebrum verticillatum. De distribuição lusoextremadurense (Rivas-Martínez et al. 1980), integrase na aliança Cicendion (Rivas Goday apud Rivas Goday \& Borja 1961) Braun-Blanquet 1967.

2. Vegetação anfíbia vivaz: classe Isoeto-Littorelletea Braun-Blanquet \& Vlieger in Vlieger 1937

Reuniu comunidades anfíbias vivazes e holárcticas (Rivas-Martínez et al. 2002) de helófitos junceiformes e isoetídeos, próprias de águas doces oligotróficas ou ligeiramente mesotróficas de níveis variáveis, paradas ou ligeiramente fluentes. Esta vegetação cresce em solos lodosos e gleiformes iniciais, meso-oligotróficos (RivasMartínez et al. 1980). Identificou-se a ordem européia Littorelletalia Koch 1926, de ótimo siberiano e reduzida representação mediterrânea ocidental (Sardinero 2004) e a aliança Hyperico elodis-Sparganion Braun-Blanquet
\& Tüxen ex Oberdofer 1957, de caráter atlântico e mediterrânico-iberoatlântico (Loidi et al. 1997).

\subsection{Associação Hyperico elodis-Rhynchosporetum} rugosae Neto, Capelo, J.C. Costa \& Lousã in Neto 1997

De distribuição sadense (Neto, C.S., dados não publicados), foi encontrada colonizando áreas com maior acumulação de água nos juncais higrófilos. No Moinho da Ordem em Alcácer do Sal (Tab. 6), dominava nos pequenos espaços por onde a água, ao escorrer, criava microcanais ou charcos. Era dominada por Hypericum elodes, Juncus bulbosus var. foliosus e Fuirena pubescens.

3. Vegetação de turfeiras planas: classe Scheuchzerio palustris-Caricetea nigrae Tüxen 1937 nom. mut. propos.

Agregou comunidades de elevada cobertura e pequeno porte, constituídas por hemicriptófitos e geófitos de caráter hidrofítico, acompanhados por numerosos briófitos, que formam, nas áreas encharcadas, turfeiras

Tabela 5. Loto subbiflori-Chaetopogonetum fasciculati.

\begin{tabular}{|c|c|c|c|c|c|c|c|}
\hline Número de ordem & 1 & 2 & 3 & 4 & 5 & 6 & 7 \\
\hline Altitude média (m) & 270 & 270 & 270 & 270 & 270 & 270 & 270 \\
\hline Área mínima (m²) & 4 & 2 & 2 & 2 & 4 & 4 & 4 \\
\hline Grau de cobertura (\%) & 100 & 90 & 95 & 100 & 100 & 100 & 100 \\
\hline \multicolumn{8}{|c|}{ Características da associação e das unidades superiores } \\
\hline Juncus bufonius & 2.3 & 1.2 & 3.2 & 1.2 & 3.2 & 2.2 & 3.2 \\
\hline Chaetopogon fasciculatus & 2.3 & 1.2 & 2.2 & 1.2 & 1.2 & 3.2 & 3.3 \\
\hline Solenopsis laurentia & 2.3 & 1.2 & 2.2 & 2.1 & 2.2 & 2.2 & 2.1 \\
\hline Kickxia cirrhosa & 2.2 & 1.2 & 2.3 & 2.1 & 1.2 & 1.1 & 1.1 \\
\hline Illecebrum verticillatum & 2.2 & 1.2 & 1.2 & 1.1 & 2.2 & 1.2 & 1.2 \\
\hline Juncus capitatus & 2.2 & - & 2.2 & 2.2 & 2.2 & 1.2 & 2.2 \\
\hline Exaculum pusillum & 1.2 & 1.2 & 1.2 & 2.1 & 1.1 & - & 1.1 \\
\hline Isolepis cernua & 1.1 & - & 1.2 & 3.2 & 3.2 & 1.2 & 1.1 \\
\hline Juncus pygmaeus & 1.2 & 2.2 & 2.1 & 2.2 & 2.2 & - & - \\
\hline Juncus hybridus & 1.2 & 1.2 & 2.2 & 1.2 & - & - & 1.1 \\
\hline Hypericum humifusum & 1.1 & - & - & 1.2 & 1.1 & 1.1 & +.1 \\
\hline Isolepsis pseudosetacea & 2.2 & - & 2.2 & 3.2 & 3.2 & - & - \\
\hline Radiola linoides & 1.2 & - & 1.1 & - & - & 1.2 & 2.2 \\
\hline Juncus tenageia & 1.2 & - & - & - & 1.2 & 2.2 & 2.2 \\
\hline Pulicaria paludosa & 2.2 & - & - & - & - & 1.2 & 1.1 \\
\hline Lotus subbiflorus & - & - & 2.2 & - & 2.2 & - & 2.3 \\
\hline Radiola linoides & +.1 & - & 1.2 & - & - & - & - \\
\hline Cicendia filiformis & - & - & - & 1.2 & - & - & - \\
\hline Myosotis caespitosa & - & - & - & - & 1.1 & - & - \\
\hline \multicolumn{8}{|c|}{ Outras espécies } \\
\hline Serapias strictiflora & - & - & 1.2 & 1.2 & 2.2 & - & 2.2 \\
\hline Pinguicula lusitanica & - & - & 1.2 & 3.2 & 2.1 & - & - \\
\hline Serapias perez-chiscanoi & - & - & - & 1.1 & +.1 & - & - \\
\hline Ranunculus trilobus & - & - & - & 1.1 & +.1 & - & - \\
\hline Silene laeta & - & - & - & - & +.1 & - & 1.1 \\
\hline Serapias lingua & - & - & - & - & 1.2 & - & - \\
\hline Serapias cordigera & - & - & - & - & +.1 & - & - \\
\hline
\end{tabular}

Locais: 1 a 7 Monte das Cabras (Vila Nova da Baronia). 
Tabela 6. Hyperico elodis-Rhynchosporetum rugosae.

\begin{tabular}{|c|c|c|c|c|c|c|c|}
\hline Número de ordem & 1 & 2 & 3 & 4 & 5 & $6^{*}$ & $7 *$ \\
\hline Altitude média (m) & 20 & 20 & 20 & 20 & 20 & 65 & 10 \\
\hline Área mínima $\left(\mathrm{m}^{2}\right)$ & 2 & 2 & 2 & 2 & 2 & 5 & 4 \\
\hline Grau de cobertura $(\%)$ & 70 & 90 & 90 & 100 & 80 & 70 & 75 \\
\hline \multicolumn{8}{|c|}{ Características da associação e das unidades superiores } \\
\hline Hypericum elodes & 2.2 & 2.1 & 2.2 & 1.2 & 3.2 & 3.2 & 3.2 \\
\hline Juncus foliosus & 1.2 & 2.2 & 1.2 & 2.3 & 1.2 & 2.2 & 1.2 \\
\hline Fuirena pubescens & 1.2 & 1.2 & 2.2 & 1.2 & 2.2 & 1.2 & +.1 \\
\hline Baldellia ranunculoides & 2.2 & 2.1 & 1.2 & 2.2 & - & - & - \\
\hline Eleocharis multicaulis & - & 1.2 & 2.2 & 2.2 & 1.2 & 2.2 & 1.2 \\
\hline Drosera intermedia & - & - & - & - & 1.2 & - & 1.2 \\
\hline Rhynchospora rugosa & - & - & - & - & - & 1.2 & 2.2 \\
\hline Hydrocotyle vulgaris & - & - & - & - & - & +.1 & 1.1 \\
\hline Utricularia exoleta & - & - & - & - & - & +.2 & +.2 \\
\hline Ludwigia palustris & - & - & - & - & - & - & 1.1 \\
\hline \multicolumn{8}{|c|}{ Outras espécies } \\
\hline Juncus acutiflorus & 2.2 & 1.2 & - & 2.3 & 1.2 & +.2 & +.2 \\
\hline Cicendia filiformis & 1.2 & 1.2 & 1.2 & - & 1.2 & - & - \\
\hline Juncus tenageia & 2.3 & 2.3 & - & 1.2 & - & - & - \\
\hline Juncus rugosus & - & 1.2 & - & - & - & +.2 & +.2 \\
\hline Anagallis tenella & - & - & 1.2 & - & 2.2 & - & - \\
\hline Carex demissa & - & - & 1.2 & - & 1.2 & +.2 & - \\
\hline Hypericum undulatum & - & - & - & 1.2 & 1.1 & +.1 & - \\
\hline Agrostis stolonifera & - & - & - & - & - & +.2 & +.2 \\
\hline
\end{tabular}

* Dados secundários, obtidos de Neto, C.S. (dados não publicados). Taxa presentes uma vez: I. F. 1 Juncus bufonius 1.2; I. F. 2 Molinia arundinacea 2.2; Schoenus nigricans 1.2; Solenopsis laurentia 1.2; I. F. 3 Lobelia urens +.2; Myosotis welwitschii +.1; I. F. 5 Sphagnum auriculatum 1.2; Agrostis stolonifera 1.1; I. F. 6 Alisma plantago-aquatica 1.1; Lycopus europaeus +.1; I. F. 7 Panicum repens +.1. Locais: 1 a 5 Moinho da Ordem; 6 Texugueiras; 7 Açude dos Cachopos (Alcácer do Sal).

baixas e planas (Rivas-Martínez et al. 2002). São de ótimo boreo-alpino holárctico (Sardinero 2004). Foi identificada a ordem Caricetalia nigrae Koch 1926 nom. mut. propos., de distribuição holárctica (Rivas-Martínez et al. 2002), e a aliança Anagallido tenellae-Juncion bulbosi BraunBlanquet 1967, de caráter atlântico (Loidi et al. 1997).

\subsection{Associação Anagallido tenellae-Juncetum bulbosi Braun-Blanquet 1967}

Esta associação foi amostrada nas turfeiras planas, sendo dominada por hemicriptófitos de pequeno porte e grande variedade de briófitos, em solos oligotróficos higro-turfosos e com nível freático elevado (Neto, C.S., dados não publicados). Essa associação foi encontrada no Açude da Batalha e Moinho da Ordem em Alcácer do Sal (Tab. 7) e era dominada por Drosera intermedia, Anagallis tenella e Sphagnum auriculatum. De acordo com Loidi et al. (1997), é de distribuição atlântica européia e mediterrânica ibérica ocidental.

4. Comunidades higrófilas de Utricularia: classe Utricularietea intermedio-minoris Pietsch 1965

De acordo com Rivas-Martínez et al. (2002), agrupa comunidades holárcticas de Utricularia L., que colonizam pequenos charcos com solos gleizados, cobertos por água de pequena profundidade, durante praticamente todo o ano. A ordem Utricularietalia intermedio-minoris Pietsch 1965 e a aliança Sphagno-Utricularion Müller \& Görs 1960 desta classe são também holárcticas (Loidi et al. 1997).

\subsection{Associação Utriculario exoletae-Sphagnetum} auriculati Neto, Capelo, J.C. Costa \& Lousã 1996

Foi identificada em turfeiras constituídas por comunidades de elevada cobertura, em áreas quase ou permanentemente cobertas por uma fina camada de água pobre em cálcio e com fraca oscilação de nível freático. Nas estações de Alcácer do Sal (Tab.8), aparecia de forma pontual nas orlas e clareiras de juncais higrófilos e encontrava-se dominada pelas insetívoras Utricularia gibba subsp. exoleta (Açude da Batalha e Moinho da Ordem) e Utricularia australis (Texugueiras). Sua distribuição é sadense (Neto, C.S., dados não publicados).

\section{Vegetação vivaz mesofítica: classe Molinio- Arrhenatheretea Tüxen 1937}

Reúne comunidades de prados densos e juncais vivazes mesofíticos e higrófilos, antropozoonitrófilos, de cobertura muito elevada, em solos profundos mais ou menos úmidos, raramente submersos, constituídos por

Tabela 7. Anagallido tenellae-Juncetum bulbosi.

\begin{tabular}{|c|c|c|c|c|c|c|c|c|c|}
\hline Tún & 1 & 2 & 3 & 4 & 5 & 6 & 7 & 8 & 9 \\
\hline n) & 29 & 29 & 29 & 29 & 22 & 22 & 20 & 20 & 22 \\
\hline &, 5 & 0,5 & 1 & & 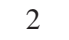 & & & ? & 1 \\
\hline & 100 & 100 & 100 & 100 & 80 & 80 & 90 & 80 & 90 \\
\hline \multicolumn{10}{|c|}{ Características da associação e das unidades superiores } \\
\hline rosera intermedia & 2.2 & 2.2 & 3.3 & 2.2 & 3.3 & 2.1 & 3.3 & 3.2 & 2.2 \\
\hline & 2.1 & 2.2 & 1.1 & 2.2 & +.2 & 1.2 & - & - & 1.1 \\
\hline & 3.3 & 3.3 & 3.2 & 2.3 & _ & - & 2.2 & 2.3 & - \\
\hline & 2.1 & 1.1 & 1.1 & 1.2 & - & - & . & & \\
\hline & & - & - & & - & +.2 & 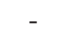 & - & 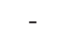 \\
\hline 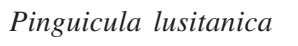 & - & - & - & - & - & - & +.1 & - & - \\
\hline \multicolumn{10}{|c|}{ Outras espécies } \\
\hline & 1.2 & 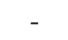 & - & +.2 & 1.2 & 1.2 & 1.2 & 1.2 & 1.2 \\
\hline & 2.2 & 2.2 & 2.2 & 3.3 & - & - & - & - & 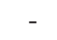 \\
\hline & & - & - & - & 1.2 & +.2 & 1.2 & 1.2 & - \\
\hline Ele & - & - & - & - & 2.3 & 2.2 & - & - & 3.2 \\
\hline Jun & - & - & - & - & 1.2 & 1.2 & - & - & 2.2 \\
\hline Fuirena & - & - & - & - & 1.2 & 1.2 & - & - & 2.2 \\
\hline Molini & - & - & - & - & 1.2 & - & 2.2 & 1.2 & - \\
\hline Jun & - & - & - & - & 1.2 & 1.2 & - & - & - \\
\hline & - & - & - & - & & - & - & 1.2 & - \\
\hline Hype & - & - & - & - & +.2 & - & - & - & 1.2 \\
\hline Myrica galle & - & - & - & - & +.2 & - & - & - & +.2 \\
\hline
\end{tabular}

Taxa presentes uma vez: I. F. 5 Lobelia urens +.1; I. F. 6 Juncus bufonius 1.2; Cicendia filiformis +.2; I. F. 7 Hypericum undulatum +.2. Locais: 1 a 4 Açude da Batalha; 5 a 9 Moinho da Ordem (Alcácer do Sal). 
Tabela 8. Utriculario exoletae-Sphagnetum auriculati.

\begin{tabular}{|c|c|c|c|c|c|c|c|c|c|c|}
\hline Número de ordem & 1 & 2 & 3 & 4 & 5 & 6 & 7 & 8 & 9 & 10 \\
\hline Altitude média (m) & 29 & 29 & 29 & 65 & 65 & 65 & 150 & 150 & 150 & 150 \\
\hline Área mínima $\left(\mathrm{m}^{2}\right)$ & 0,5 & 0,5 & 0,5 & 1 & 1 & 1 & 1 & 1 & 1 & 1 \\
\hline Grau de cobertura $(\%)$ & 80 & 80 & 80 & 80 & 80 & 100 & 80 & 90 & 100 & 90 \\
\hline \multicolumn{11}{|c|}{ Características da associação e das unidades superiores } \\
\hline Sphagnum auriculatum & 2.2 & 1.2 & 2.2 & 1.2 & 1.2 & 2.2 & - & - & 2.2 & 1.2 \\
\hline Utricularia exoleta & 2.2 & 2.2 & 2.1 & 1.2 & +.2 & +.1 & - & - & - & - \\
\hline \multicolumn{11}{|c|}{ Outras espécies } \\
\hline Drosera intermedia & 2.2 & 1.2 & 2.2 & 3.2 & 2.3 & 2.2 & 1.1 & - & 1.2 & - \\
\hline Anagallis tenella & 1.2 & - & 1.2 & - & 1.2 & 2.2 & 1.2 & 1.1 & - & 1.2 \\
\hline Isoetes hystrix & - & 1.2 & 2.2 & 2.2 & 2.2 & 2.2 & - & - & - & - \\
\hline Eleocharis multicaulis & - & 1.2 & - & - & - & - & 2.2 & 1.2 & 2.2 & 2.2 \\
\hline Fuirena pubescens & - & 1.2 & - & - & - & - & +.2 & 1.2 & +.2 & 1.2 \\
\hline Hypericum elodes & - & 1.1 & - & - & - & - & 1.2 & - & - & 1.2 \\
\hline Isoetes setacea & - & - & - & - & - & - & 2.2 & 2.2 & 1.2 & - \\
\hline Schoenus nigricans & - & +.2 & - & - & +.2 & - & - & - & - & - \\
\hline Potentilla erecta & - & - & - & 1.2 & 1.2 & - & - & - & - & - \\
\hline Juncus rugosus & - & - & - & - & - & - & +.2 & +.1 & - & - \\
\hline
\end{tabular}

Taxa presentes uma vez: I. F. 4 Molinia arundinacea +.2; I. F. 6 Pinguicula lusitanica 3.2; Carex demissa +.1; I. F. 9 Hypericum humifusum 1.2; Juncus sorrentini 1.1; Agrostis stolonifera +.2; I. F. 10 Juncus foliosus 1.1; Potamogeton natans 1.1. Locais: 1 a 3 Açude da Batalha; 4 a 6 Moinho da Ordem; 7 a 10 Texugueiras (Alcácer do Sal).

plantas vivazes, na sua maioria hemicriptófitos e criptófitos, que, em algumas ocasiões, podem ser exploradas pelo homem (Pereira, M.M.D., dados não publicados). Identificou-se a ordem Molinietalia caeruleae Koch 1926, que é eurossiberiana, mas pode ocorrer no ocidente da Região Mediterrânica (Loidi et al. 1997). A aliança Juncion acutiflori Braun-Blanquet in Braun-Blanquet \& Tüxen 1952 aparece nas áreas de influência atlântica e mediterrânica-iberoatlântica (Loidi et al. 1997).

\subsection{Associação Cirsio palustris-Juncetum rugosi Neto, Capelo, J.C. Costa \& Lousã 1996}

Foi identificada em juncais higrófilos dominados por hemicriptófitos, que colonizavam os leitos de alguns ribeiros turfosos, encharcados, submersos durante o Inverno. Essa associação foi amostrada no Açude da Batalha, em Alcácer do Sal (Tab. 9), nos espaços ocupados no passado com orizicultura e, hoje, abandonados. Era dominada por Festuca arundinacea, Molinia caerulea subsp. arundinacea e Juncus acutiflorus subsp. rugosus. Encontra-se no Setor Ribatagano-Sadense e Superdistrito Sadense (Neto, C.S., dados não publicados).

As comunidades anfíbias do sudoeste de Portugal, em particular do Estuário do Rio Sado, estão inseridas num ambiente tipicamente mediterrânico, com precipitação anual inferior a $600 \mathrm{~mm}$ e um prolongado período seco, com temperaturas elevadas. Dessa forma, é surpreendente a ocorrência de comunidades vegetais com elevada frequiência de plantas com distribuição
Tabela 9. Cirsio palustris-Juncetum rugosi.

\begin{tabular}{|c|c|c|c|c|c|c|}
\hline Número de ordem & 1 & 2 & 3 & 4 & $5^{*}$ & $6^{*}$ \\
\hline Altitude média (m) & 29 & 29 & 29 & 29 & 45 & 68 \\
\hline Área mínima $\left(\mathrm{m}^{2}\right)$ & 8 & 8 & 8 & 8 & 15 & 12 \\
\hline Grau de cobertura $(\%)$ & 100 & 100 & 100 & 100 & 100 & 100 \\
\hline \multicolumn{7}{|c|}{ Características da associação e das unidades superiores } \\
\hline Festuca arundinacea & 2.3 & 2.2 & 2.2 & 2.3 & - & +.2 \\
\hline Molinia arundinacea & 2.2 & 2.2 & 3.3 & 2.2 & 3.2 & 3.2 \\
\hline Juncus rugosus & - & 1.2 & +.2 & 1.2 & 2.2 & 2.2 \\
\hline Schoenus nigricans & 1.2 & +.2 & - & - & - & - \\
\hline Holcus lanatus & - & - & - & - & 2.2 & +.2 \\
\hline Juncus effusus & - & - & - & - & +.2 & +.2 \\
\hline Cirsium palustre & - & - & - & - & 1.1 & 1.1 \\
\hline Lythrum junceum & - & - & - & - & +.1 & +.1 \\
\hline Galium palustre & - & - & - & - & 1.1 & +.1 \\
\hline Oenanthe lachenalii & - & - & - & - & +.1 & - \\
\hline Carex lamprocarpa & - & - & - & - & 2.2 & - \\
\hline Lotus pedunculatus & - & - & - & - & +.2 & - \\
\hline \multicolumn{7}{|c|}{ Outras espécies } \\
\hline Sphagnum auriculatun & $m 2.3$ & $2.2^{1}$ & 2.3 & 2.3 & - & - \\
\hline Anagallis tenella & 1.2 & 1.1 & 1.2 & 2.1 & - & - \\
\hline Isoetes setacea & 2.2 & - & 2.2 & 2.2 & - & - \\
\hline Potentilla erecta & 1.2 & - & 1.1 & 2.1 & +.1 & - \\
\hline Drosera intermedia & +.2 & - & +.2 & +.1 & - & - \\
\hline Eleocharis multicaulis & - & 2.3 & 2.2 & 2.2 & - & - \\
\hline Fuirena pubescens & - & 1.2 & 1.2 & 2.2 & - & - \\
\hline Lythrum salicaria & - & - & - & - & 1.1 & 1.1 \\
\hline Genista ancistrocarpa & - & - & - & - & +.1 & 1.1 \\
\hline Ulex minor & - & - & - & - & +.1 & +.1 \\
\hline
\end{tabular}

* Dados secundários, obtidos de Neto, C.S. (dados não publicados). Taxa presentes uma vez: I. F. 5 Schoenus nigricans 1.2; Carex lusitanica 1.2; Lotus uliginosus +.2 ; Phragmites australis +.1; I. F. 6 Schoenoplectus mucronatus 4.2; Hypericum elodes 2.1; Hydrocotyle vulgaris 1.1; Rhynchospora rugosa +.2 ; Rubus ulmifolius +.1 . Locais: 1 a 4 Açude da Batalha; 5 Apostiça; 6 Texugueiras (Alcácer do Sal). 
atlântica e necessidades hídricas. Na verdade, em todo o sudoeste de Portugal, as comunidades vegetais anfíbias de elevados teores de umidade estão associadas a depressões em substratos arenosos, sobrepostos a materiais mais duros e compactos, que travam o movimento descendente da água de gravidade. A extensa toalha freática que se forma na base das areias surge à superfície nas depressões, constituindo espaços úmidos de características palustres ou turfosas, com presença de água durante todo o ano. É essa original circulação hidrológica que permite a existência de comunidades vegetais com características atlânticas em pleno mundo mediterrânico: "ilhas atlânticas em ambiente mediterrânico".

Considerada a composição florística, verificou-se a presença de inúmeras plantas com uma evidente disjunção relativamente à sua área de distribuição contínua no Noroeste da Península Ibérica e Portugal, de clima atlântico, como Myrica galle, Cirsium palustre, Sphagnum auriculatum e Pinguicula lusitanica. Em relação à vegetação, muitas das comunidades vegetais que ocorreram na área estudada apresentaram-se isoladas em pequenas bolsas e, portanto, são relativamente raras. Correspondem a uma vegetação relictual, que registrou o seu ótimo no período Atlântico (7800-5700 B.P.), durante o Quaternário, quando o clima mais úmido permitiu a migração, de norte para sul, desse tipo de flora.

No atual quadro de alterações climáticas (verões progressivamente mais quentes e precipitações concentradas num período de tempo cada vez menor), a área ocupada pelas comunidades estudadas tem-se reduzido de forma significativa, encontrando-se algumas delas em perigo de extinção. A principal causa de destruição dos hábitats dessas comunidades vegetais tem sido, no entanto, o impacto antrópico (exploração agrícola e florestal e os empreendimentos turísticos). Dessa forma, o interesse que apresentam para proteção e conservação é elevado e, por isso, estão incluídas na Rede Natura 2000, algumas em regime de proteção especial.

\section{Agradecimentos}

Os autores agradecem à Profa. Dra. Marlene Durigan, da Universidade Federal de Mato Grosso do Sul, pela disponibilidade em rever o texto apresentado.

\section{Referências bibliográficas}

Aedo, C. \& Herrero, A. (eds.). 2005. Flora Iberica. Plantas vasculares de la Península Ibérica e Islas Baleares. v.XXI - SmilacaceaeOrchidaceae. Madrid, Real Jardín Botánico, CSIC.

Barkman, J.J.; Moravec, J. \& Rauschert, S. 1988. Código de Nomenclatura Fitosociológica. Opuscula Botanica Pharmaciae Complutensis 4: 5-74.
Barros e Carvalhosa, A. \& Galopim de Carvalho, A.M. 1969. Notícia Explicativa da folha 40-A (Viana do Alentejo) da Carta Geológica de Portugal na escala 1/50 000. Lisboa, Serviços Geológicos de Portugal.

Braun-Blanquet, J. \& Tüxen, R. 1952. Irische Pflanzengesellschaften. Veröff. Ber. Geobot. Inst. E.T.H. Stiffung Rübel 25: 222-421.

Braun-Blanquet, J. 1936. Über die Trockenrasengesellschaften des Festucion vallesiacae in den Ostalpen. Bulletin de la Société Botanique Suisse 46: 169-189.

Braun-Blanquet, J. 1967. Vegetationsskizzen aus dem Baskenland mit Ausblicken auf das weitere Ibero-Atlantikum. II Teil. Vegetatio 14: 1-126.

Braun-Blanquet, J. 1979. Fitosociologia. Bases para el estudio de las comunidades vegetales. Madrid, Ed. Blume.

Brullo, S. \& Minissale, P. 1998. Considerazione sintassonomiche sula classe Isoeto-Nanojuncetea. Itinera Geobotánica 11: 263-290.

Castroviejo, S.; Aedo, C.; Laínz, M.; Morales, R.; Muñoz Garmendia, F.; Nieto Feliner, G. \& Paiva, J. (eds.). 1997 b. Flora Iberica. Plantas vasculares de la Península Iberica e Islas Baleares. v.V - Ebenaceae-Saxifragaceae. Madrid, Real Jardín Botánico, CSIC.

Directiva 92/43/CEE do Conselho de 21 de maio de 1992. (Anexo II). Jornal Oficial das Comunidades Europeias N. L 206/7 de $22 / 7 / 92$.

Franco, J.A. \& Afonso, M.L.R. 1994, 1998, 2003. Nova Flora de Portugal (Continente e Açores). v.III (fasc. I, II, III) Lisboa, Escolar Editora.

Franco, J.A. 1971, 1984. Nova Flora de Portugal (Continente e Açores). v.I, II. Lisboa, Ed. do Autor.

Géhu, J.M. \& Rivas-Martínez, S. 1981. Notions fondamentales de Phytosociologie. Pp. 1-33. In: J. Cramer (ed.). Syntaxonomie. Vaduz, Ber. Internat. Symp. IVVS.

Guinochet, M. 1973. Phytosociologie. Paris, Masson e Cie. Éditeurs.

Izco, J. \& Del Arco, M. 2003. Código internacional de nomenclatura fitosociológica. Materiales Didácticos Universitarios. Serie Botánica 2: 13-154.

Koch, W. 1926. Die Vegetationseinheiten der Linthebene, Nordostschweiz. Jb. St. Gallischen Naturwiss. Ges. 61: $1-134$.

Loidi, J.; Galarraga, I.B. \& Gallastegui, M.H. 1997. La vegetación del centro-septentrional de España. Itinera Geobotánica 9: $161-618$

Moor, M. 1937. Ordnung der Isoetetalia (Zwergbinsengesellschaften). Prodrome des Groupements Végétaux 4: 1-189.

Müller, T. \& Görs, S. 1960. Pflanzengesellschaften stehender Gewässer in Baden-Württemberg. Beitr. Naturk. Forsch. Südwestdeutschl 19: $60-100$.

Neto, C.S. 1997. A flora e a vegetação dos meios palustres do Superdistrito Sadense. Lisboa, Centro de Estudos Geográficos.

Neto, C.S.; Capelo, J.H.; Costa, J.C. \& Lousã, M. 1996. Sintaxonomia das Comunidades de turfeira do Superdistrito Sadense. Silva Lusitana 4: 257-258.

Oberdofer, E. 1957. Süddeutsche Pflanzengesellschaften. Jena, Gustav Fischer Verlag.

Pereira, M.M.D. 2003. Método fitossociológico de BraunBlanquet ou clássico sigmatista (análise da vegetação). Évora, Departamento de Planeamento Biofísico e Paisagístico. Universidade de Évora.

Pietsch, W. 1965. Utricularietea intermedio-minoris class. nov. Ein Beitrag zur Klassifizierung der europäischen WasserschlauchGesellschaften. Berichte der Arbeitsgemeinschaft Sächsischer Botaniker 5/6: 227-231.

Rivas Goday, S. \& Borja, J. 1961. Estudio de la vegetación y flórula del Mácizo de Gúdar y Javalambre. Anales del Instituto Botánico A. J. Cavanilles 19: 1-550.

Rivas Goday, S. 1954. Comunidades de la Nanocyperion flavescentis W. Koch en Extremadura. Anales del Instituto Botánico A. J. Cavanilles 12: 443-467. 
Rivas Goday, S. 1957. Nuevos órdenes y alianzas de Helianthemetea annuae Br. B1. Anales del Instituto Botánico A.J. Cavanilles 15: $539-651$.

Rivas Goday, S. 1958. Bases ecológicas y estadísticas de la Fitosociología. Anales de la Real Academia Nacional de Farmacia 24: 191-210.

Rivas-Martínez, S.; Costa, S.; Castroviejo, S. \& Valdés, E. 1980 Vegetación de Doñana (Huelva, España). Lazaroa 2: 5-190.

Rivas-Martínez, S.; Díaz, T.E., Fernández-González, F.; Izco, J.; Loidi, J.; Lousã, M. \& Penas, A. 2002. Vascular Plant Communities of Spain and Portugal. Addenda to the syntaxonomical checklist of 2001. Itinera Geobotánica 15: 433-922.

Rivas-Martínez, S.; Fernández-González, F.; Loidi, J.; Lousã, M. \& Penas, A. 2001. Syntaxonomical Checklist of Vascular Plant Communities of Spain and Portugal to Association Level. Itinera Geobotánica 14: 5-341.

Sardinero, S. 2004. Flora y vegetación del macizo occidental de la Sierra de Gredos (Sistema Central, España). Guineana 10: $5-474$.
Tüxen, R. 1937. Die Pflanzengesellschaften Nordwestdeutschlands. Mitteilungen der Floristisch-Soziologischen Arbeitsgemeinschaft 3: $1-170$.

Tutin, T.G.; Heywood, V.H.; Burges, N.A.; Moore, D.M.; Valentine, D.H.; Walters, S.M. \& Webb, D.A. (eds.). 1964, 1968, 1972, 1976, 1980. Flora Europaea. v.I, II, III, IV, V. 2 ed. Cambridge, Cambridge University Press.

Valdés, B.; Talavera, S. \& Galiano, F. (eds.). 1987. Flora vascular de Andalucia Occidental. v.I, II, III. Barcelona, Ketres Editora S.A.

Vlieger, J. 1937. Aperçu sur les unités phytosociologiques supérieures des Pays-Bas. Ned. Kruidk. Arch. 47: 335-353

Westhoff, V.; Dijk, J.W. \& Passchier, H. 1946. Overzicht der plantengemeenschappen in Nederland (Overview of the plant communities in the Netherlands). Amsterdan, B.W. Breughel.

Zbyszewski, G.; Antunes, M.T. \& Ferreira, O.V. 1976. Notícia explicativa da folha 39-A (Águas de Moura) da Carta Geológica de Portugal na escala 1/50 000. Lisboa, Serviços Geológicos de Portugal. 